Itinéraires Itinéraires

Littérature, textes, cultures

2020-2 | 2020

Discours animal. Langages, interactions, représentations

\title{
Les affichettes d'animaux perdus. Discursivité, agentivité, anthroponymie
}

Lost Pet Posters. Discursivity, Agency, Anthroponymy

\section{Elsa Eskenazi et Marie-Anne Paveau}

\section{(2) OpenEdition}

\section{Journals}

Édition électronique

URL : https://journals.openedition.org/itineraires/8637

DOI : $10.4000 /$ itineraires.8637

ISSN : 2427-920X

Éditeur

Pléiade

Référence électronique

Elsa Eskenazi et Marie-Anne Paveau, « Les affichettes d'animaux perdus. Discursivité, agentivité, anthroponymie », Itinéraires [En ligne], 2020-2 | 2020, mis en ligne le 22 décembre 2020, consulté le 24 novembre 2021. URL : http://journals.openedition.org/itineraires/8637 ; DOI : https://doi.org/10.4000/ itineraires.8637

Ce document a été généré automatiquement le 24 novembre 2021.

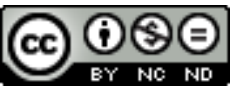

Itinéraires est mis à disposition selon les termes de la licence Creative Commons Attribution - Pas d'Utilisation Commerciale - Pas de Modification 4.0 International. 


\title{
Les affichettes d'animaux perdus. Discursivité, agentivité, anthroponymie
}

Lost Pet Posters. Discursivity, Agency, Anthroponymy

\author{
Elsa Eskenazi et Marie-Anne Paveau
}

\section{Introduction}

1 Cette contribution propose un format hybride entre récit d'expérience, biographie d'objets et analyse écologique du discours. Le travail se fonde sur un jeu de données de 32 éléments sélectionnés dans une collection de 194 affichettes d'animaux perdus recueillies en contexte urbain, dans des villes françaises et étrangères, entre 2015 et 2018. La sélection complète est disponible en ligne ${ }^{1}$.

2 Nous écrivons à deux, à partir de positions et de représentations différentes. La série d'affichettes appartient à Elsa Eskenazi, ancienne comédienne, qui a effectué son recueil en collectionneuse; en ethnologue spontanée, elle en fait ici l'histoire, en explique les objectifs et l'intérêt, et met au jour les ressorts subjectifs de sa collection. Marie-Anne Paveau en examine les dispositifs énonciatifs et sémiotiques avec un regard d'universitaire spécialiste d'analyse du discours, impliquée dans la mise en place d'une linguistique non dualiste, ouverte sur les règnes animaux et végétaux (une analyse écologique du discours).

3 L'ensemble forme un texte qui n'a pas pour objectif d'entrer tout à fait dans le format universitaire, mais qui se présente plutôt comme une note de recherche sous forme de description organisée de matériaux empiriques, intégrant plusieurs types de savoirs, profanes et savants. La première partie, rédigée par Elsa Eskenazi, est consacrée à la description subjective de la collection; la deuxième, consacrée aux affichettes en tant qu'objets, et la troisième, dédiée à l'agentivité discursive des animaux représentés, sont 
écrites par Marie-Anne Paveau. Chacune parle en première personne; quand un nous apparaît, il désigne la réflexion commune.

\section{Ethnographie spontanée d'une collection}

\subsection{La collection}

4 Je suis devenue collectionneuse il y a environ 5 ans.

Je collectionne certains types d'objet: les réveils cassés, les affichettes d'animaux, les pelles usées, les clés et les "photos de tas variés» que je prends dès que j'en ai l'occasion.

Toutes mes collections sont collaboratives et sont soumises à des règles strictes.

Si j'achète des réveils cassés ils doivent coûter au maximum 5 euros.

Les pelles c'est 8 euros.

Les affichettes doivent être arrachées dans la rue, sans autorisation.

Tout le monde a le droit de collaborer à mes collections. J'ai appelé mes collaborateurs les Agents de terrain.

5 Les Agents de terrain sont soumis aux mêmes règles et ont un avenir tout tracé. Celui qui m'apportera le plus d'éléments héritera de la collection qu'il a fait grandir avec moi (à ce jour certains d'entre eux tremblent et regrettent déjà. Tant pis).

6 Je récupère, j'arrache, je reçois, je prends, je négocie ou j'achète. Puis j'entasse, j'amasse, je dispose, j'expose ou je range les trésors dans mon appartement. Tout ça en sautillant joyeusement bien sûr.

7 Mes collections, c'est comme ma toile de fond, mon arrière-monde. Un mur très rassurant contre lequel je m'appuie. Un mur porteur. C'est une zone vivante. Une sorte de verbalisation d'une mythologie intérieure. On cohabite gaiement. Elles sont un langage à part entière selon moi. Un discours, un rébus, qui travaille, sans moi, avec moi, malgré moi. Il y a de l'impensé dans mes collections et ce flou est volontaire. Il y a le plaisir immense d'accumuler. Un geste qui me guérit.

On pourrait définir une collection comme un motif qui se décline à l'infini. C'est aussi une promesse que je scelle je ne sais pas bien où ni avec qui. Mes affects s'y fixent. Plus ou moins fort.

Les affichettes d'animaux sont sans hésitation une de mes collections préférées.

\subsection{Les affichettes d'animaux perdus}

10 Maintenant mes yeux ont appris et savent.

Mes yeux - c'est vrai et il faut le dire - sont des radars à affichettes. Je sais parfaitement où elles sont, où elles seront, où on les mettra.

Mes yeux scannent la ville à la recherche de cette recherche.

11 Les murs, les coins, les poteaux électriques, les entrées et sorties de métro, les abribus, certains types d'arbres deviennent des lieux de message sans adresse.

Des correspondances en forme de bouteille à la mer. À l'attention des attentifs ou des concernés. Il y a de l'espoir. C'est un appel aux passants. Une interpellation. Alors moi oui je m'arrête. 
Entre 2015 et 2018 j'ai récolté 194 affichettes. Autant dire un vrai petit tas d'or.

Il y a ceux qui cherchent l'animal «chat/chien perdu » (173 affichettes) et ceux qui cherchent l'humain propriétaire «chat/chien trouvé» (14 affichettes), ceux qui accusent de vol (" animal volé », 3 affichettes) et ceux qui ont perdu des oiseaux et rêvent qu'une occasion quasi impossible se présente pour les récupérer ( 4 affichettes d'oiseaux envolés par la fenêtre, dont la perruche ci-dessous).

Figure 1

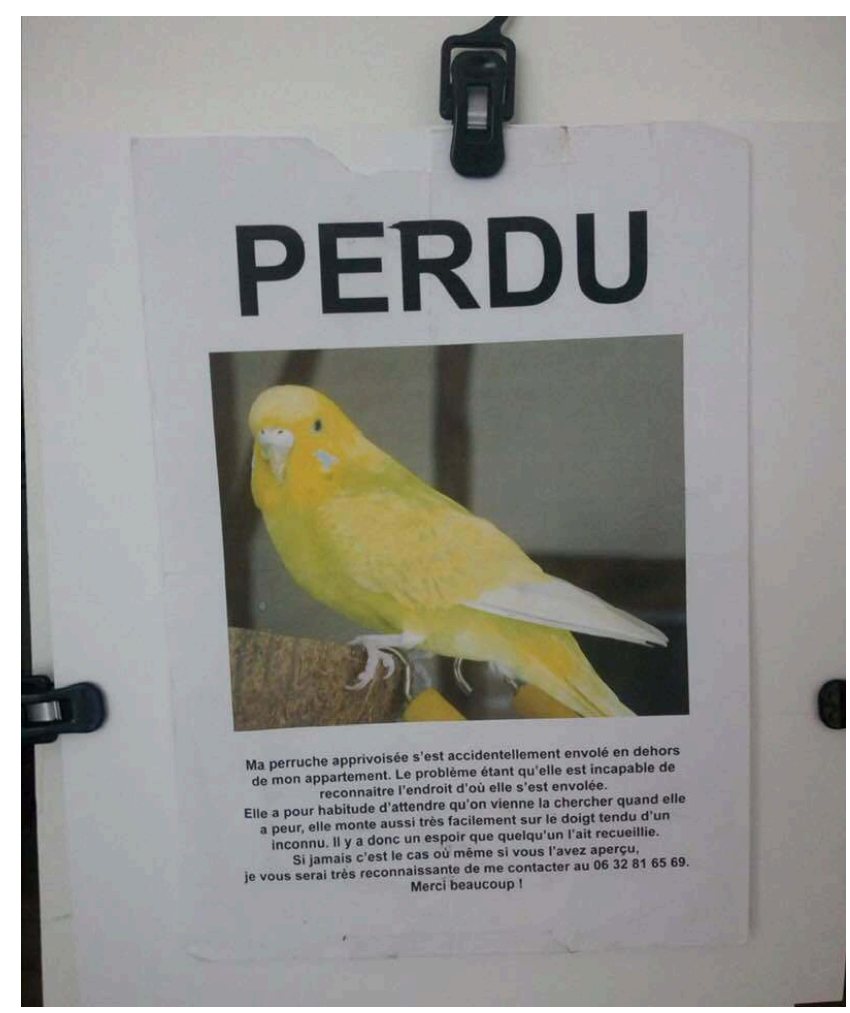

«Elle a pour habitude d'attendre qu'on vienne la chercher quand elle a peur. Elle monte aussi très facilement sur le doigt tendu d'un inconnu. II y a donc un espoir que quelqu'un l'ait recueillie. »

Moi, disons-le franchement, je suis toujours du côté du perdu. C'est vrai que je ne fais pas de distinction entre l'animal perdu, trouvé ou volé. Quand je découvre un chat dans mon jardin, cela me renvoie tout de suite au jardin où le chat n'est plus. Donc peu importe le choix sur le papier, l'affichette est pour moi une affaire de perte, car si ce chat a été trouvé c'est bien qu'il a été perdu pour d'autres. Le fameux verre à moitié vide ou à moitié plein ; sur les questions d'affichettes mon verre est toujours à moitié vide.

Quand on farfouille dans ma collection on peut voir un tas d'animaux perdus sur du papier évidemment, mais aussi l'empreinte de mes pas. On peut déduire dans quelle ville ou quel arrondissement j'ai vécu par exemple ces quatre dernières années.

Cette collection est liée à la ville. Elle se fabrique avec le déplacement piéton quel qu'il soit et où qu'il soit : l'errance, les trajets quotidiens et répétitifs, la promenade légère, les hasards, les détours, les imprévus, les voyages.

Ces animaux appartiennent désormais aux villes et quand j'arrache leur affichette, j'arrache avec elle un bout de territoire et un morceau de temps. 
16 découverte de l'animal errant (124 affichettes datées). Et cette date me fascine. Cette précision me fascine. C'est rare. Ça annonce un nouveau point de départ. Ça dit que c'est important. Il y aura peut-être un avant et un après cette date. À part dans les cimetières ou sur les faire-part, je ne sais pas à quel moment on inscrit et on investit autant d'affect dans une date. J'adore tout simplement. Je ne sais pas bien pourquoi. J'adore cette importance à nommer les dates. C'est grâce à cette importance que je découvre aussi parfois que certaines affichettes ont longuement cohabité avec le quartier avant que, miraculeusement, je les découvre, les arrache et les emporte. Personne n'y a prêté attention avant moi peut-être.

Mais pourquoi le propriétaire n'a-t-il pas enlevé l'affichette? Le chat est-il mort ou revenu, a-t-il été adopté par d'autres ? Le propriétaire espère-t-il encore ? Est-ce pour cette raison qu'elle est encore là sur ce poteau? A-t-il oublié ? Remplacé son animal ? Les questions restent en suspens. On n'en saura jamais rien. Ce mystère me stresse joyeusement et m'intrigue. Mes émotions sont toujours paradoxales et emmêlées quand ça concerne les affichettes.

Parfois ces affichettes sont si bien pensées et équipées qu'elles peuvent résister à toutes les météos et à tous les mouvements de la ville. Parfois donc elles savent aussi me résister. Trop bien scotchées, trop bien collées. Si je les prends je les déchire, et, déchirées elles ne peuvent pas rentrer dans mon petit mausolée personnel. Je renonce donc. Maintenant, j'ai appris à renoncer plus vite. Je la touche et rapidement je sais que je ne pourrai pas l'avoir. Certains donnent quand même dans la surprotection du message. Incroyablement bien plastifié. Ils me font rire. Je les comprends parfaitement: je suis redoutable. D'autres sans doute moins angoissés ou moins prévoyants (ce qui peut revenir au même) laissent la ville s'imprimer dessus et cela donne au papier une texture incroyable et très particulière que je manipule avec beaucoup de soin. Comme un trésor fragile (18 affichettes au papier fragile). L'encre de l'impression se décompose en arc-en-ciel bizarre. Parfois les affiches sont tellement délavées par la pluie le vent le soleil que le message est à peine lisible ou le texte complètement effacé. Seuls les yeux du chat ressortent par exemple.

fait qu'une affichette est une affichette c'est le texte. Il y a toujours du texte, il n'y a pas toujours de date ou de photo mais il y a toujours du texte et toujours un numéro à joindre si jamais.

Parfois le message est concis net, sans fioriture (58 affichettes). Il n'est qu'informatif descriptif. Ça ne jacte pas, ça dit, ça progresse, on ne s'épanche pas, comme dans cette affichette minimaliste : 
Figure 2

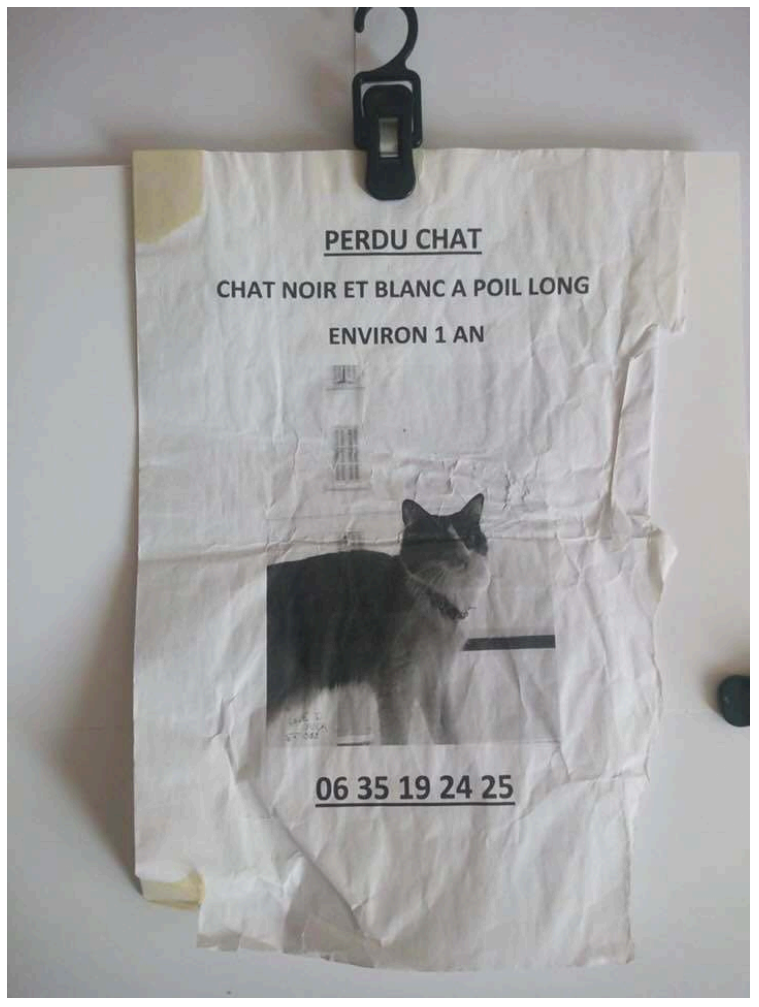

D'autres se lancent dans d'incroyables descriptions détaillées de leur animal : «Croisée rottweiler, robe noire et feu, pas de queue, collier rouge. Elle est très gentille et un peu sourde, on note à sa démarche que c'est un vieux chien. " J'imagine qu'ils craignent qu'on leur rapporte un sosie, pas la version originale perdue. Certains tentent d'appâter le piéton avec des promesses de "grosse récompense à la clé » ( 35 affichettes). Il y a ceux, très créatifs ou complètement au bout du rouleau, qui font des versions très différentes de leur affichette et inondent le quartier de leurs écrits (8 affichettes déclinées en 2 ou 3 versions, voir plus bas en 2., les exemples des chattes Dune et Julia, et du chat Charlie). Certains disent explicitement l'intensité de leur inquiétude. Ils cherchent une empathie et une adhésion efficace et rapide. Plusieurs choix s'offrent alors à eux. Dix d'entre eux jouent la carte familiale et peuvent même utiliser leurs enfants : "Aidez-nous s'il vous plaît. Il nous manque énormément. Mes enfants sont très tristes. » Quatorze disent leur détresse personnelle (« je suis très malheureux sans elle ») et sept nous expliquent la fragilité de leur animal (" apeurée désorientée par le vent, elle est de plus malvoyante » ou «ma chienne a besoin de son traitement antiépileptique »).

Mon message préféré, et il est unique, est celui qui confesse pudiquement un oubli : «Oublié samedi 23 juillet dans son sac (noir) de transport sur le trottoir [...] 》 (voir cidessous l'image de Gino en 3.2.2.).

Il avoue que c'est de sa faute. J'adore les aveux c'est vrai.

Et sur les 194, j'en possède une qui est un trésor d'humanité. Une seule où le texte est particulièrement long. Il retrace un incroyable historique des évènements avant la disparition de la petite chienne. Il dit tout l'amour, l'indéfectible attachement et le deuil impossible, inenvisageable. 
Figure 3

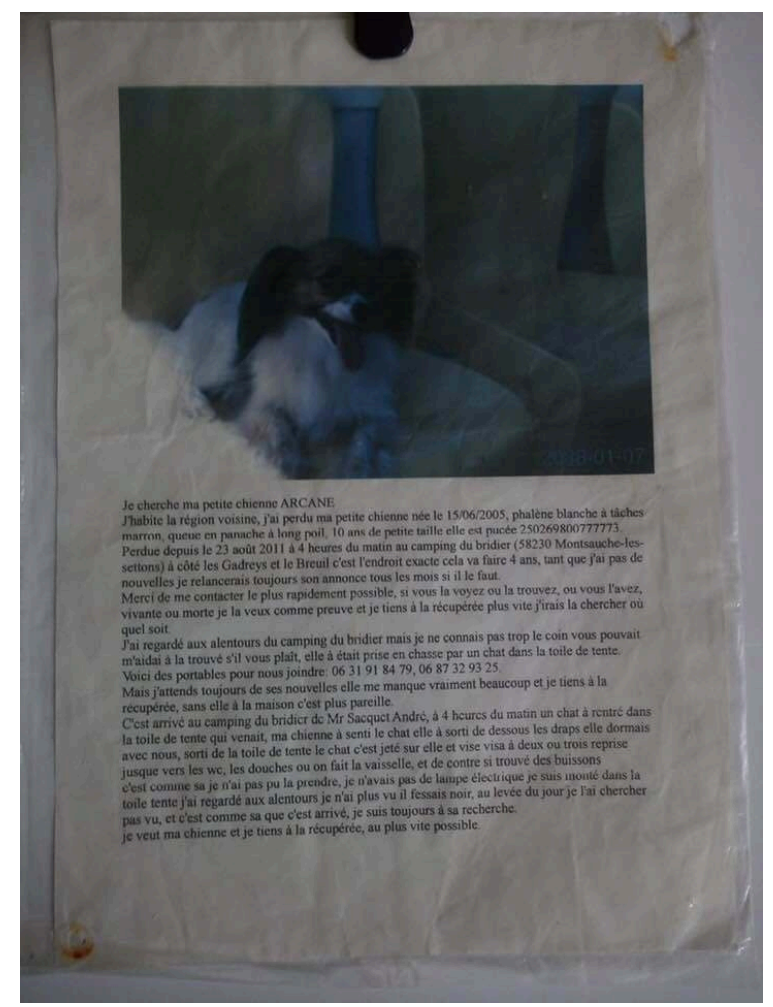

" cela va faire 4 ans, tant que j'ai pas de nouvelle je relancerais toujours son annonce tous les mois si il le faut. [...] Si vous la voyez ou la trouvez ou vous l'avez vivante ou morte je la veux comme preuve et je tiens à la récupérée plus vite j'irais la chercher où quel soit ».

Ce texte arracherait des larmes à n'importe qui tant il est un cri de détresse sincère. Cette affichette, il me semble important de le préciser également, n’a pas été prise dans une grande ville - elle vient de Bourgogne, de la campagne - ce qui dit quelque chose également. On ne fait pas le même type d'affichette, on ne tisse peut-être pas les mêmes liens avec les animaux à la campagne et dans les villes.

\subsection{Plonger dans des morceaux de vie qui ne sont pas à moi}

Mes 194 affichettes m'ont appris qu'il y a plusieurs façons de chercher ceux qu'on aime.

Après la date, la photo est ce que je préfère décortiquer. Les décors sont très variés, et le point de vue sur l'animal est toujours singulier et spécifique. La photo est comme une invitation pour moi. Un langage à part entière.

Elle me dit : «Alors? Qu'est-ce que tu peux te dire et imaginer à partir de moi ? vas-y vas-y je t'écoute."

Il y a des animaux à la plage au bistrot dans le métro sous des meubles dans des jardins sur un lit dans la rue. Ils sont rabougris dans un coin, majestueux sur un coussin, hyper-expressifs ou complètement sinistres, tenus en laisse ou gambadant librement. Certaines photos sont parfaitement absurdes et ambiguës, on reconnaît à peine l'animal mais par contre on distingue très bien le salon coquettement meublé.

Tout cela me dit des choses. Chuchote des choses dans mon oreille aiguisée.

22 J'aime ces bouts de vie humaine et animale mêlés sur le même document adressé à tout le monde, adressé pour l'entraide, adressé dans le vide des villes grouillantes ou des 
campagnes désertées. J'aime l'alliance des dates, du texte et de la photo. J'aime ce type de montage. Ça me touche.

L'affichette saisit mon présent, elle surgit sur ma route et mon cœur heureux d'avoir bien fouillé les environs se félicite puis bondit toujours dans un petit sursaut joyeux. Tout à coup la ville parle et m'apporte l'histoire d'une perte.

Avec cette irruption de réel je peux imaginer. Et ça, ça ne se discute plus. C'est ce que je préfère faire dans la vie. Imaginer des histoires.

Ce petit chat mignon a peut-être été exploité pendant des années comme une bête à concours, grâce à lui ses propriétaires se sont fait une solide petite réputation à laquelle ils s'accrochent de toutes leurs forces mais, épuisé par les déplacements et la pression incessante, écœuré par l'ambiance mortifère de l'univers des concours de beauté le chat s'est enfui. Ciao tout le monde. Aujourd'hui il est libre enfin de mener une vie de chat plus en adéquation avec ses utopies - oui c'est possible.

Avec l'affichette c'est comme si je plongeais tout entière dans des morceaux de vie qui ne sont pas à moi. J'ai une sensation de plongeon, donc de vertige également. Je n'arrive tout simplement pas à laisser ces messages seuls dans la rue. Alors oui j'arrache je prends, je vole je récolte et je consigne les pertes.

Ce n'est pas qu'emphatique comme geste, loin de là, je sais que je laisse aussi s'exprimer à travers cette collection une petite forme de cruauté personnelle ou de folie-noirâtreun-peu-grinçante-qui-fait-du-bien.

Parfois je vois un chat dans la rue et je pense tout de suite à l'affichette en devenir qu'il est en train de constituer, ou je pense à l'affichette qu'il est déjà et que je n'ai pas encore. Je suis fixée sur l'affichette. Je regarde le chat filer en me disant : «Toi et moi bientôt on se retrouvera, sois-en certain. » Lui comme tous les autres il finira classé, rangé dans mes enveloppes bien classées, bien rangées, sur l'étagère où je classe et range bien. Là-bas, là-haut, chez moi où tout est bien en ordre, où le monde est en état de marche. On sera bien.

Ça me réconforte et ça me soigne de réunir les perdus. Les animaux perdus, les messages perdus, les propriétaires perdus sans leur animal. Je les regroupe, je les garde, je les protège chez moi avec moi. Ils sont à moi. On est ensemble. On n'est plus seuls. On est tous ensemble dans l'unique monde que je comprends enfin parfaitement bien, celui de ma collection.

\section{Des objets et du sens}

Elsa Eskenazi vient de le montrer : l'objet est bien connu, surtout à la ville, mais également à la campagne. Il s'agit essentiellement de feuilles au fond blanc de format A4 ou plus petites, rarement plus grandes, parfois protégées par une pochette plastique, comprenant une ou plusieurs photos de l'animal perdu (majoritairement des chats et des chiens), un texte dactylographié (assorti parfois de notations manuscrites), des coordonnées téléphoniques, parfois une adresse électronique, un $Q R$ code. Elles sont le plus souvent scotchées sur les arbres, les poteaux télégraphiques, les horodateurs, les panneaux d'annonces des supermarchés.

La sélection des 32 affichettes s'est faite à partir du critère d'agentivité. Nous définissons l'agentivité, traduction de la notion d'agency théorisée par Judith Butler (2004), comme une puissance d'agir liée à notre capacité de langage, conçue non 
comme une faculté que nous maîtriserions mais comme une compétence à laquelle nous serions assujettiees ; l'agentivité est alors une manière d'être sujet du discours, au sein des déterminations socio-historiques qui nous façonnent. Ont été retenues les affichettes qui présentaient l'expression d'un discours animal, d'une perception animale ou d'une humanisation par le biais d'un prénom apparemment ${ }^{2}$ humain, traits pouvant constituer l'animal en sujet. La sélection comporte deux doublons, comptés pour une seule affichette (la chatte Dune, 1.5 et 1.5bis ; la chatte Julia, 3.14 et 3.14bis) : il s'agit de la même affichette, des notations manuscrites constituant une version deux. La collection comporte un cas particulier de trois affichettes concernant le même chat, mais différentes dans leur forme, à trois moments différents de la recherche, et constituant une sorte de narrativisation de la quête (Charlie, 3.6, 3.7 et 3.8); elles sont comptées comme trois affichettes distinctes.

Ces affichettes relèvent de deux catégories, que je souhaite articuler et traiter ensemble, dans la perspective écologique de ce travail : ce sont, sur le plan matériel, des objets et, sur le plan sémiotique, des phototextes relevant d'un genre de discours stabilisé.

\subsection{Des objets discursifs graphiques}

Les objets produisent du sens: dotés d'une agentivité et d'une sémioticité, ils contribuent à la production du sens en société. Je reprends ici l'une des cinq catégories proposées en 2012 dans "Ce que disent les objets. Sens, affordance, cognition" (Paveau 2012): j'y distinguais les outils linguistiques (dictionnaires, guides de correspondance), les outils discursifs (listes, post-its), les outils composites linguistiques-discursifs (ordinateurs, smartphones), les objets discursifs graphiques (bouteilles, emballages) et les objets discursifs non graphiques (verre, foulard). Ces cinq catégories d'objets contribuent chacune à leur manière à la production du discours, les objets discursifs graphiques le faisant car ils sont dotés de traits graphiques orientant les productions discursives.

Ce travail sur les affichettes d'animaux perdus constitue une sorte de biographie d'objets (discursifs graphiques), au sens où Arjun Appadurai entend cette notion: la biographie d'objet est en effet un genre qui rend compte de l'agentivité et de l'historicité de l'objet, considéré dans ses connexions relationnelles et ses itinéraires sociaux, et non plus comme une chose inerte outillant l'activité humaine ou participant juste de son décor. C'est un outil méthodologique issu de la notion de "vie sociale des objets " proposée par Arjun Appadurai dans le collectif qu'il dirige en 1986, The social life of things. Commodities in cultural perspective. Pour lui, la valeur des objets ne tient pas seulement à des critères économiques ou sociaux, mais tient aux objets eux-mêmes dans leur matérialité, aux messages qu'ils délivrent et aux actions auxquelles ils participent. Il prône l'adoption d'un «fétichisme méthodologique» pour rendre compte de ce que font véritablement les objets dans la vie sociale :

Ainsi, même si, d'un point de vue théorique, les acteurs humains confèrent leurs significations aux choses, d'un point de vue méthodologique ce sont les choses-enmouvement qui éclairent leur contexte humain et social. Aucune analyse sociale des choses (que l'analyste soit un économiste, un historien de l'art ou un anthropologue) ne peut éviter un niveau minimum de ce que l'on pourrait appeler $\mathrm{du}$ fétichisme méthodologique. Si nous tournons à nouveau notre attention vers les choses elles-mêmes, ce fétichisme méthodologique apporte un correctif partiel à la 
tendance de sociologiser à l'excès les transactions sur les choses, tendance que nous devons à Mauss, ainsi que Firth l'a noté naguère. (Appadurai [1986] 2009 : 4)

C'est Lorraine Daston qui introduit ensuite la méthode de la biographie d'objet dans le collectif qu'elle dirige en 2000, Biographies of Scientific Objects (Daston ed. 2000), dans le cadre d'une "métaphysique appliquée » qui conteste la division traditionnelle entre perception et réalité et voudrait que l'histoire des sciences ne soit pas seulement une histoire de ce qui est connu, mais également de ce qui est. Accorder une agentivité aux objets c'est, comme le disent Antoine Hennion et Bruno Latour, revenir sur l'antifétichisme dominant dans les sciences humaines et sociales et cesser de penser que les objets ne sont que des projections de la société : «Les objets font quelque chose, et d'abord ils nous font » (Hennion et Latour 1993 : 9).

Les objets sont donc des producteurs de signes et des contributeurs à la production des discours. Cela siginifie que l'ordre du non-humain objectal coconstruit les discours dans l'environnement global de notre vie, et que nous parlons donc dans les objets. Les affichettes d'animaux perdus, inscrites dans le paysage scriptural et plus largement sémiotique de la ville, jouent un rôle non seulement comme porteuses de signes, mais aussi comme objets connus, reconnaissables, avec leur pochette plastique parfois un peu sale, leurs morceaux de scotch de tous types, leurs photos stéréotypées. Elles ont été photographiées sans souci esthétique, avec le désir de conserver leurs caractéristiques matérielles: déchirures, salissures, morceaux de scotch, pochettes plastique abimées, etc. Elles sont simplement maintenues sur un carton blanc avec une pince visible sur la photo ${ }^{3}$.

3 Sur les six affichettes ci-dessous, le système d'accrochage est utilitaire : le ruban adhésif d'emballage avec sa classique couleur brune (1.3 et 2.4) ou en version verte issue de plastique recyclé de marque Tesay (3.6.) ou même l'adhésif de réparation argenté (3.14bis), comme le scotch transparent classique collé avec des plis (3.1 et 3.23), semblent surtout destinés à faire tenir le plus solidement l'affichette, sans souci d'esthétique. Dans tous les cas le scotch est un élément visible et même saillant de l'affichette. Il fait partie du stéréotype de l'affichette d'animal perdu, et c'est un trait matériel du genre. 
Figure 4. Affichette 1.3. Jasper

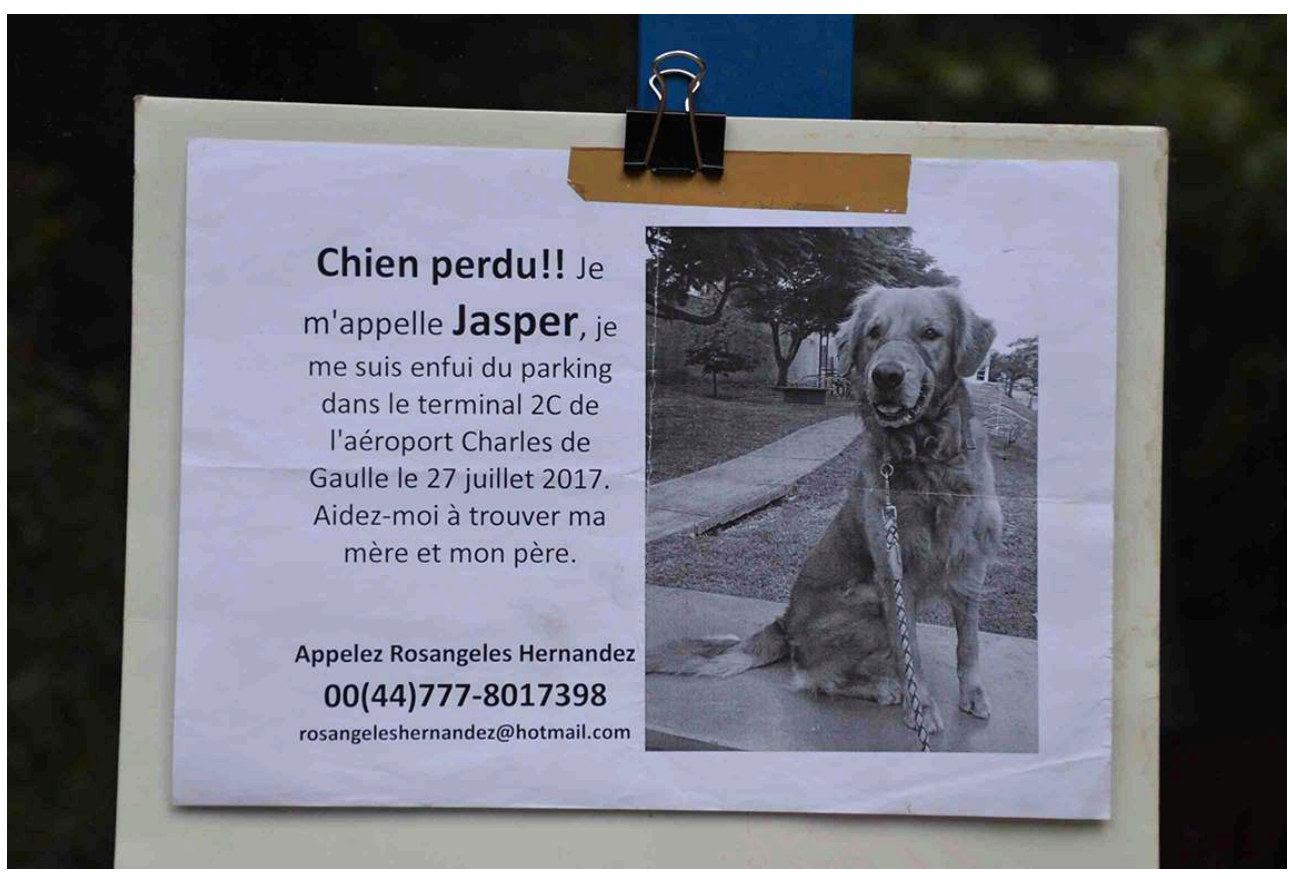

Figure 5. Affichette 2.3. Simone

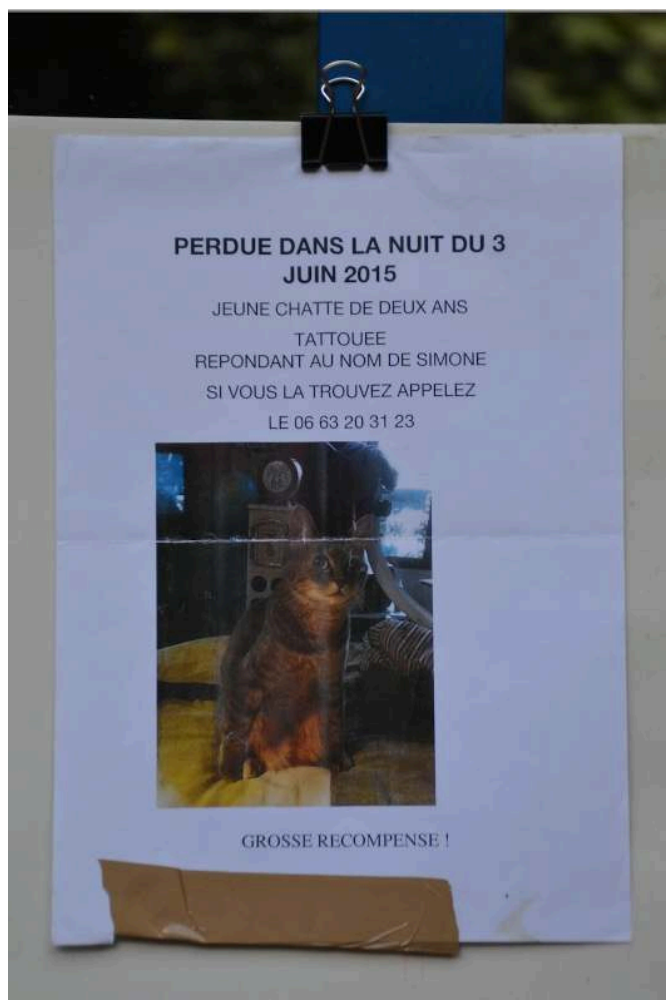


Figure 6. Affichette 3.1. Freddy

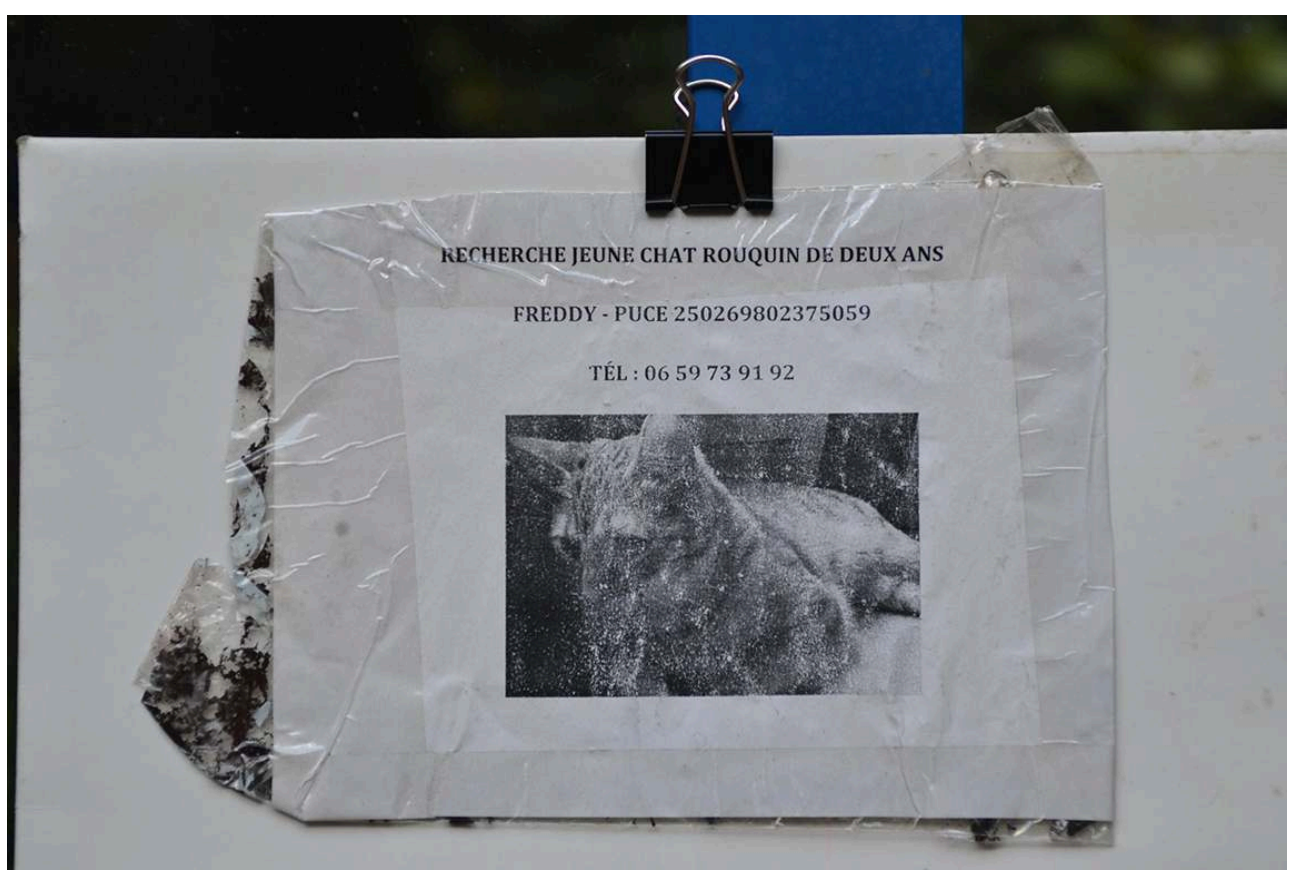

Figure 7. Affichette 3.6. Charlie

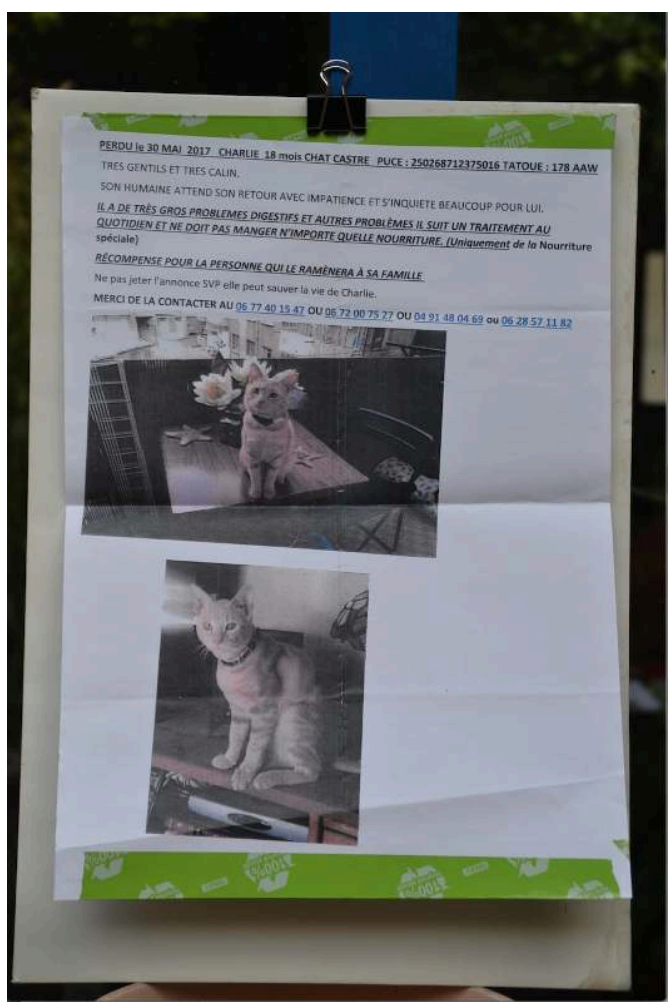


Figure 8. Affichette 3.14bis Julia

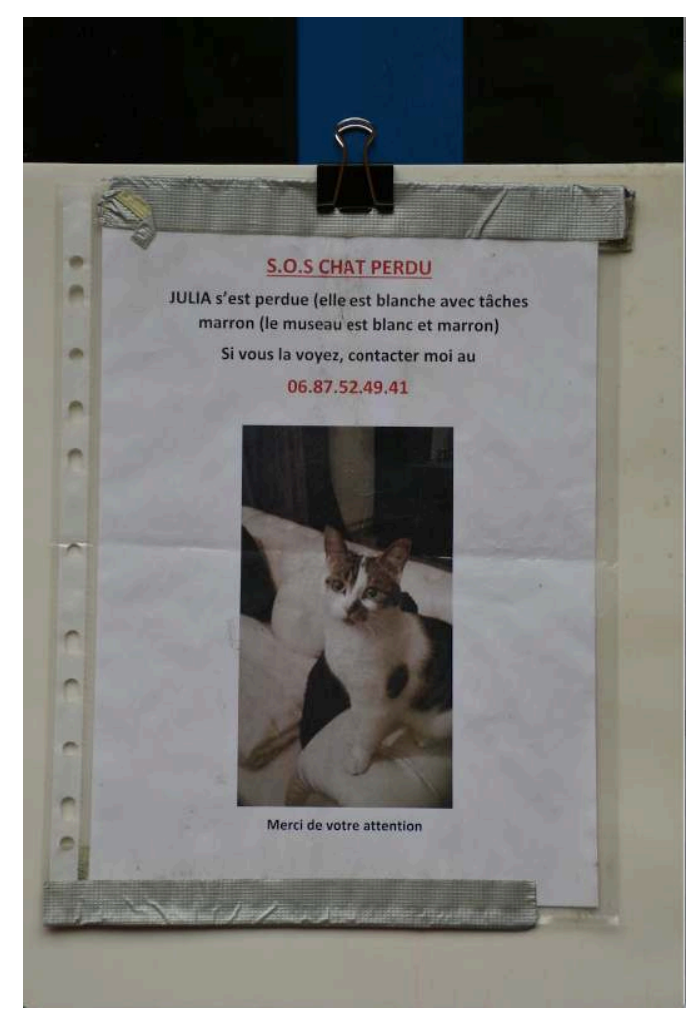

Figure 9. Affichette 3.23 Diego

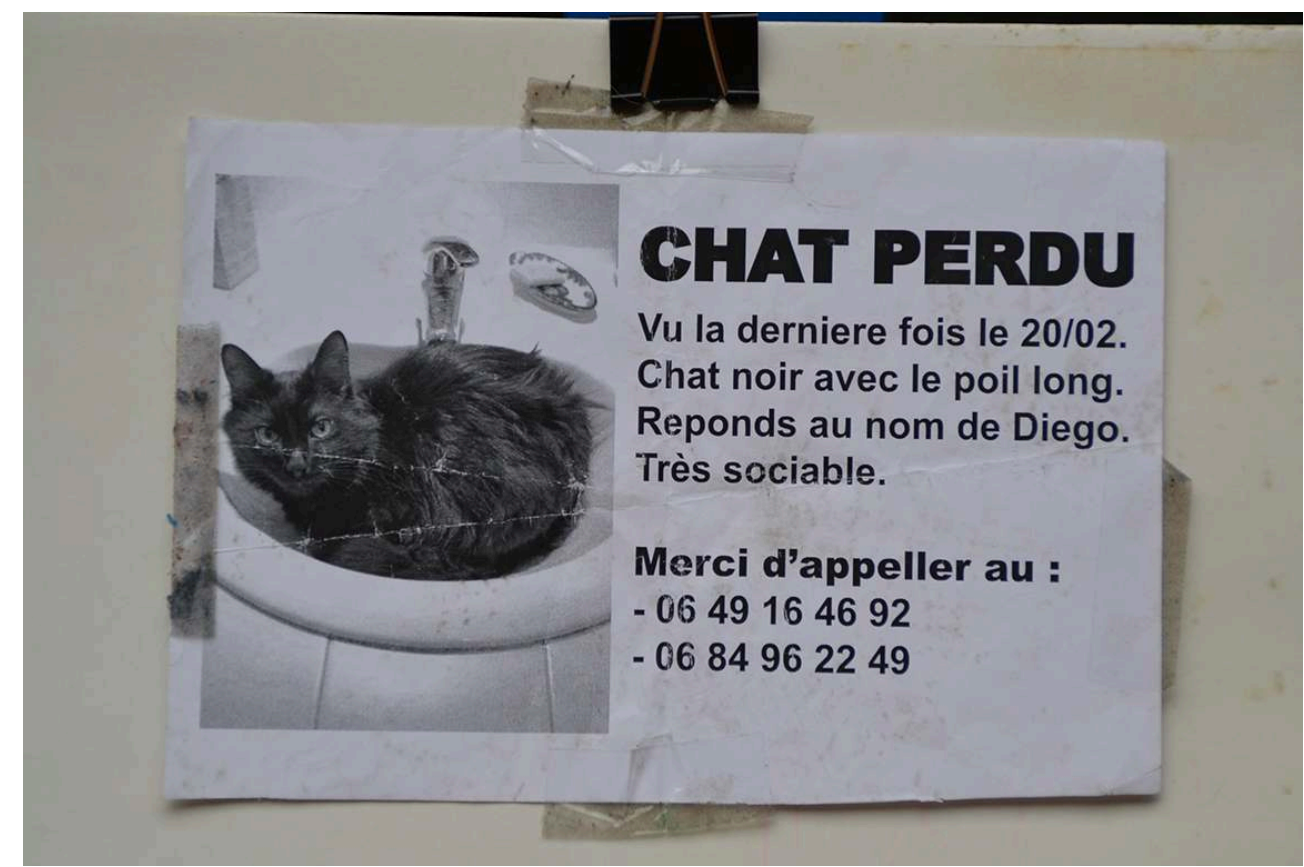

\subsection{Des phototextes stéréotypés}

Les affichettes sont des formes plurisémiotiques relevant d'une composition entre le texte et l'image. J'appelle énoncé composite (Paveau 2017, article «Composition») un 
énoncé présentant une articulation structurelle entre le texte et l'image, aucun des deux éléments ne pouvant fonctionner de manière autonome, la construction du sens se faisant en un seul temps dans l'articulation et non en deux temps (le texte constituant la légende de l'image ou l'image l'illustration du texte). On peut alors parler de phototexte (Stafford 2010), terme que je préfère à celui d'iconotexte, plus connu, proposé par Michael Nerlich en 1990 pour rendre compte d'un mode d'articulation entre le texte et l'image qui laisse leur autonomie aux deux composants. Dans l'affichette d'animal perdu, cette autonomie n'existe pas et les deux éléments forment, par composition, une unité de sens.

Ces composites sont stéréotypés car ils présentent des traits formels et sémantiques communs, qui les rendent reconnaissables par les récepteur.trice's. Comme on le voit aisément sur les éléments de la collection, et comme on le sait sans doute également, par notre expérience de la lecture d'images dans la ville européenne, cinq éléments sont récurrents :

- le mot-clé perdu, décliné sous différentes formes et dans différents formats syntaxiques (chat perdu, SOS chat perdu, perdu le, chien perdu !!, perdue ou volée, nous avons perdu, missing, etc.) ;

- la date et éventuellement le lieu de la disparition ;

- la ou les photographie(s) en couleur ou noir et blanc, selon le type de photocopie ;

- le texte du ou de la propriétaire de l'animal ;

- ses coordonnées téléphoniques et parfois électroniques.

Ce stéréotype constitue un genre, au sens où l'entend la linguistique du texte ou du discours, c'est-à-dire un modèle reproductible, reconnaissable, modifiable et transmissible, inscrit dans les compétences cognitives des individus. En ce qui concerne nos affichettes, la stabilité du genre est assurée notamment par de nombreux modèles en ligne, qui permettent de fabriquer des affichettes en remplissant des catégories préconstruites. Sur le site Alerte Animaux perdus.fr par exemple ${ }^{4}$, un modèle d'annonce, qui permettra de générer une page pdf imprimable, propose de renseigner le nom, la race, la couleur, l'âge, le sexe, le tatouage, la puce, la présence d'un collier et sa couleur. L'internaute peut déposer un message, télécharger jusqu'à 4 photos. Il renseigne ensuite le lieu de la disparition, la date et ses coordonnées et peut ajouter un QR code que les passantees pourront flasher pour accéder directement à l'annonce du site. Il y a trois modèles possibles: chien, chat, autre. L'affichette imprimable est également partageable sur Facebook et Twitter. De nombreux autres sites présentent le même type de service, qu'il s'agisse de sites dédiés aux animaux perdus ou d'espaces plus largement dédiés à la rédaction. Sur le site REP Secrétariat (REP pour Rigueur, Efficacité, Professionnalisme $)^{5}$, on trouve la proposition suivante : 


\section{Voici 2 modèles d'affiches à télécharger gratuitement via la boutique pour vous aider dans la recherche de votre chat ou de votre chien :}

2 affiches par page, à découper et à coller partout :

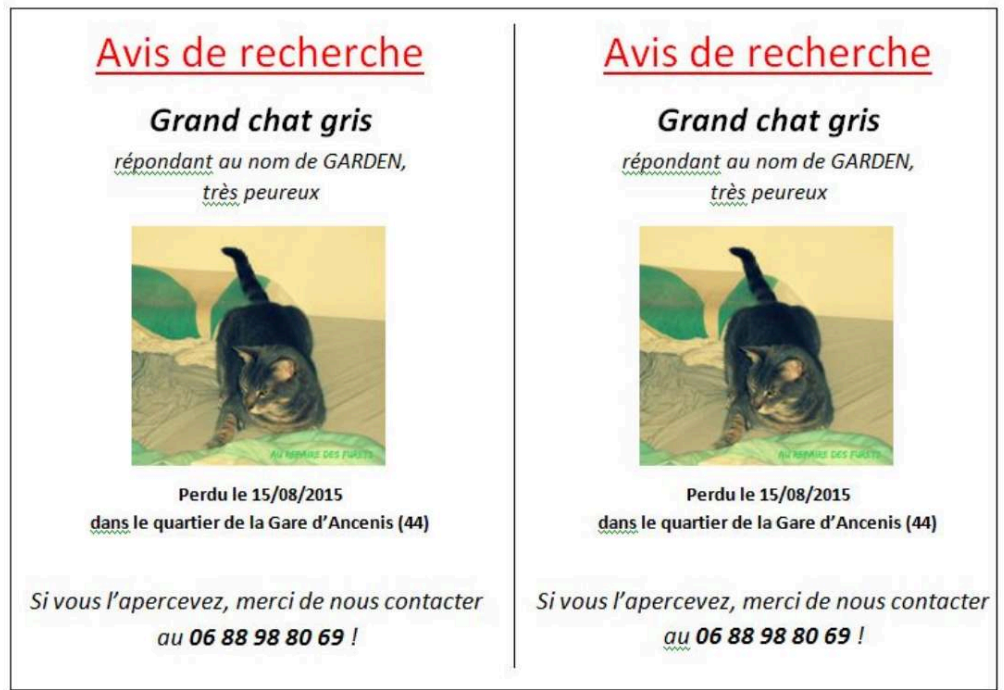

Sur le site Chat perdu, une page de conseils pour fabriquer une affichette ${ }^{6}$ reprend ces éléments, en ajoutant des conseils sur les pochettes plastiques pour protéger les affichettes de la pluie, sur les lieux d'affichage, sur le nombre d'affichettes à préparer. Mais deux conseils en particulier sont intéressants pour notre étude. Le premier : «Il ne faut pas non plus en dire trop pour que l'affichette reste très visuelle ", va dans le sens d'une primauté sémantique de l'image sur le texte dans la construction du sens, ce qui relève de ce que Williams J. Thomas Mitchell appelle le pictorial turn de la communication : l'image pilote le sens (Mitchell 1994). Le second: « De nouveau, il est inutile d'être trop précis sur le lieu de la disparition, cela ne servira à rien. La seule présence de l'affichette à un endroit suffit pour dire qu'un animal a été perdu à proximité ", indique que l'affichette d'animal perdu est déictique ; cela veut dire que l'affichette est directement référentielle, sur le plan du lieu, elle contient en elle-même les coordonnées spatiales de la disparition. Ces deux traits de l'affichette, sa puissance iconique et sa déicticité, en font des objets particulièrement producteurs de sens, de véritables contributeurs à la production humaine du discours.

\section{Les animaux, sujets de leur existence}

Cette agentivité de l'affichette comme objet est doublée d'une agentivité des animaux, entrée dans les sciences humaines et sociales grâce aux travaux de biologistes et philosophes au tournant des $\mathrm{xIX}^{\mathrm{e}}$ et $\mathrm{xx}^{\mathrm{e}}$ siècle, en particulier Jacob Von Uexküll. 


\subsection{Environnement et agentivité}

Dans Les Origines animales de la culture, Dominique Lestel retrace précisément cette entrée de l'agentivité animale dans l'épistémologie des sciences humaines, entrée qui signe selon lui la naissance des "sciences de l'animal ", en consacrant un chapitre à "L'animal comme sujet ». Il y montre comment le célèbre concept de monde animal ou monde propre (Umwelt) de Jakob von Uexküll, biologiste et philosophe allemand, élaboré notamment à partir du non moins célèbre exemple de la tique, a contribué à détacher l'animal de la conception cartésienne de l'animal-machine pour en faire un animal-sujet (Uexküll [1934] 1965) : «Les animaux sont composés d'organes et non de parties comme les machines. Un sujet les anime et non un moteur. L'ensemble des organes constitue un concert qui conduit la tonalité vivante de l'animal en entier. Quand cette tonalité disparaît, l'animal meurt» (Lestel 2001 : 243). Outre Jakob von Uexküll, le psychologue néerlandais Frederik Buytendijk a également fait avancer la cause de l'animal-sujet, en montrant dans son Traité de psychologie animale (Buytendijk 1952) que le comportement de l'animal permet d'accéder à sa subjectivité : «Le comportement nous apprend à connaître à la fois l'animal et son univers. Nous pouvons utiliser ce moyen d'accéder à l'univers interne de l'animal en vertu de ce que Buytendijk appelle une "universelle affinité et parenté" des attitudes de l'humain et de celles de l'animal » (Lestel 2001 : 250). Pour Frederik Buytendijk, les animaux n'ont pas de réaction mécanique aux choses du monde, mais en réalisent une interprétation :

L'animal est sujet parce qu'il pratique un mode d'existence qui place sa réceptivité aux significations intelligibles en même temps qu'il crée lui-même ces significations auxquelles il réagit de façon intelligente. L'animal est donc une «subjectivité regardée » pour Buytendijk. Pour lui, nous appréhendons spontanément l'animal comme un sujet, et nous nous appuyons d'ailleurs sur de bonnes raisons pour le faire. Il nous est en effet difficile de ne pas considérer que l'animal est un sujet alors que nous jugeons que son comportement est intelligent et qu'il agit en fonction de la signification qu'il attribue à certains éléments de son environnement. Le comportement de l'animal devient ainsi l'expression de son activité intentionnelle. (Lestel $2001: 252$ )

C'est ce dont témoignent les affichettes d'animaux perdus : du regard porté sur eux par leurs humain'e-s. Ces productions verbales constituent des représentations, des enregistrements même, voire des preuves de l'agentivité animale : l'anthropomorphisme dont elles témoignent n'est pas un simple phénomène émotionnel ou un jeu esthétique, mais une véritable entrée dans le monde animal. Renvoyer le fait que les animaux y parlent et soient dotés de traits, en particulier anthroponymiques, en principe réservés aux humain'ess, à une fonction anthropomorphe relèverait de ce que Frans De Waal appelle l'anthropodéni (De Waal 2018) : la négation de la ressemblance entre les animaux et les humain·es, servant à maintenir la distinction voire l'opposition entre les deux règnes, et à reléguer l'animal au niveau de la chose inerte, de manière à ce que la supériorité humaine reste garantie. Tout au contraire, l'anthropomorphisme dit la proximité des règnes, l'humanité de l'animal rencontrant l'animalité de l'humaine. C'est ce regard sur l'animal qui est l'objet de la catégorisation et de l'analyse qui suit. 


\subsection{Typologie discursive de l'agentivité}

39 À partir du jeu de données des 32 affichettes, a été constitué un petit corpus ${ }^{7}$ selon un critère d'agentivité décroissante repérable en discours. Par agentivité, on entend une autonomie cognitive et comportementale des animaux, incluant la production de parole. Par "répérable en discours ", on entend présent dans des segments langagiers (du type emploi de la première personne ou anthroponyme humain). Les affichettes sont classées selon trois catégories construites en fonction du degré décroissant d'autonomie énonciative et agentive des animaux :

- Discursivité animale. Appartiennent à cette catégorie les affichettes présentant des animaux dotés d'une parole autonome et produisant leur propre discours.

- Agentivité animale. Dans cette catégorie se trouvent les affichettes présentant des animaux auxquels leurs propriétaires attribuent une agentivité.

- Anthroponymie animale. Sont intégrées dans cette catégorie les affichettes présentant des animaux prénommés de manière anthroponymique (Blanchard 2015, Coulmont 2016).

Les trois catégories peuvent évidemment se croiser, mais pour des raisons pratiques les croisements ne sont pas pris en compte ici.

\subsubsection{Discursivité animale}

Sur les 32 affichettes, 5 contiennent un discours en première personne de l'animal perdu. Outre le chien Jasper, qui figure plus haut (affichette 1.3.), les chats et chattes Ellie, Sancho et Dune, et la chienne Kaye parlent sur leurs affichettes :

Figure 11. Affichette 1.1. Ellie

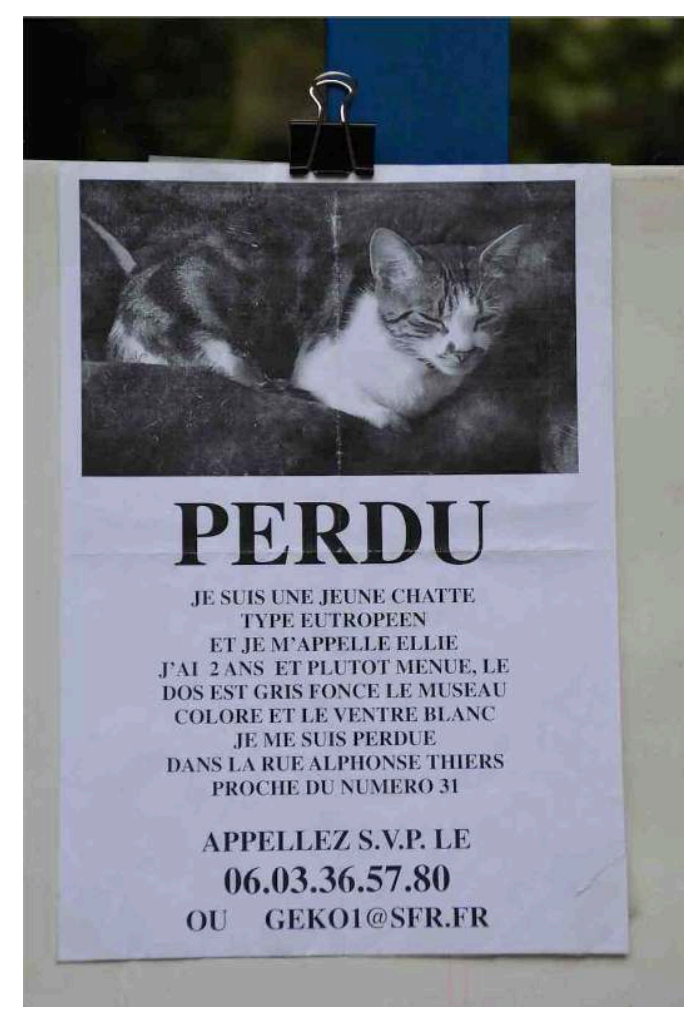


Figure 12. Affichette 1.2. Kaye

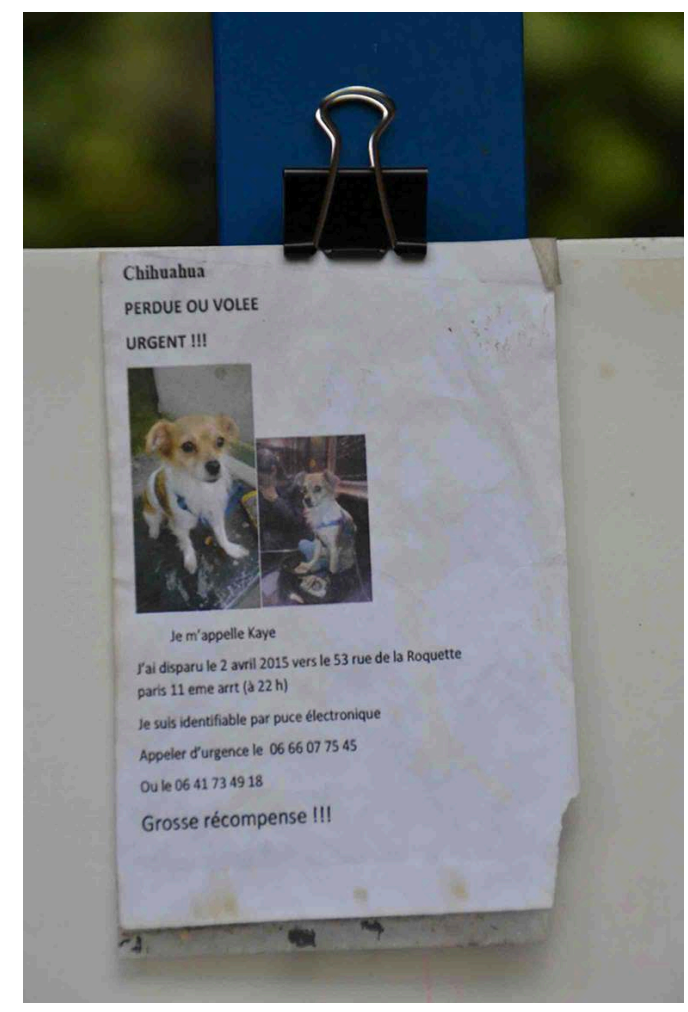

Figure 13. Affichette 1.4. Sancho

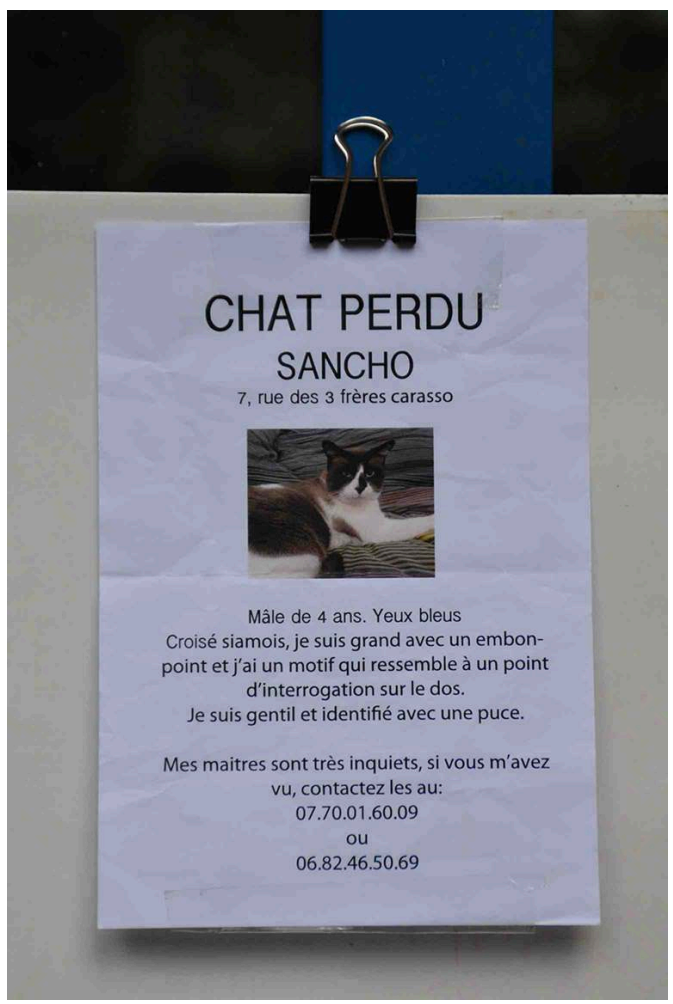


Figure 14. Affichette 1.5. Dune

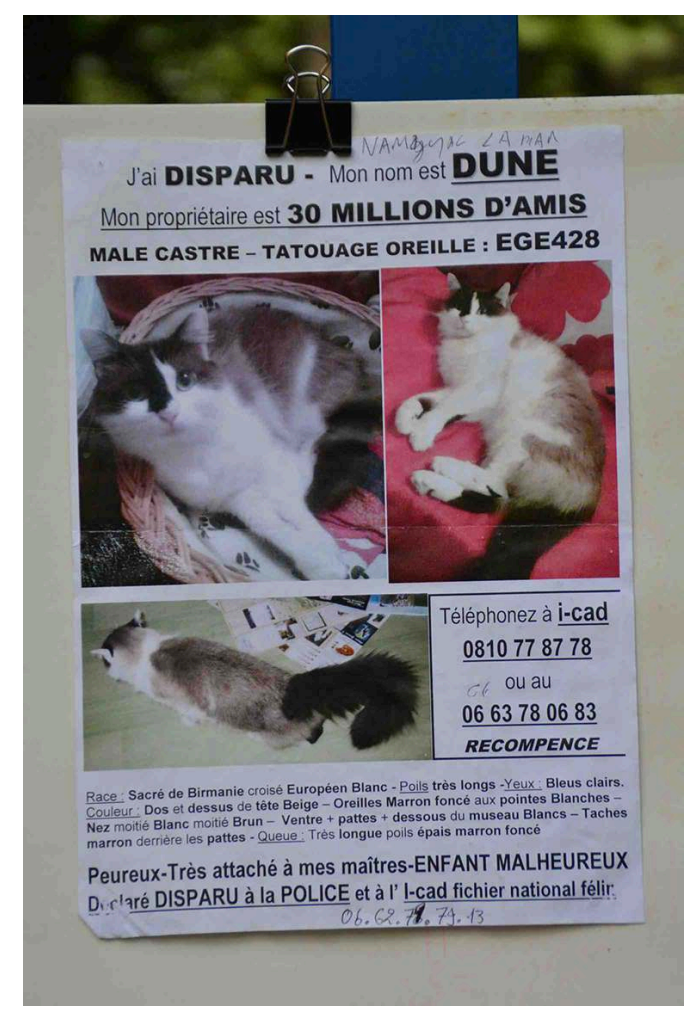

41 Que disent les animaux? Ils disent leur nom, le lieu et la manière dont ils se sont perdus (« je me suis enfui », dit Jasper), se décrivent, parfois très précisément (Dune), parlent de l'attachement de leurs maîtres (Sancho et Dune), ou de leur " mère et père " (Jasper). L'expression de ce lien affectif entre les animaux et leurs humain'e's est sans doute le point qui rend le mieux compte de la réalité de la perception animale regardée par les humaine's. Ces dispositifs énonciatifs ne sont donc pas tant des figures de style que la formulation des capacités communicatives des animaux; d'une discursivité animale.

\subsubsection{Agentivité animale}

42 Une deuxième catégorie d'affichette contient des marques d'agentivité animale, c'est-àdire d'autonomie comportementale. Sur les 32 affichettes de la sélection, 3 présentent ce trait. Outre Simone, présentée plus haut (affichette 2.3), les chats Hermi et Gino sont sujets de certains verbes qui impliquent une manière de vivre. Hermi "habite " au 47 rue Lepic ; Gino «serait heureux de rentrer rapidement à la maison pour retrouver sa petite sœur »; et de manière plus faible, agentivement parlant, Simone « répond » à son nom, avec l'ambiguïté du verbe (répondre au nom de signifie aussi « s'appeler »). Ce type d'agentivité, moins forte que la discursivité, nous semble cependant jouer le même rôle : rendre compte de l'autonomie et de la perception animales. 
Figure 15. Affichette 2.1. Hermi

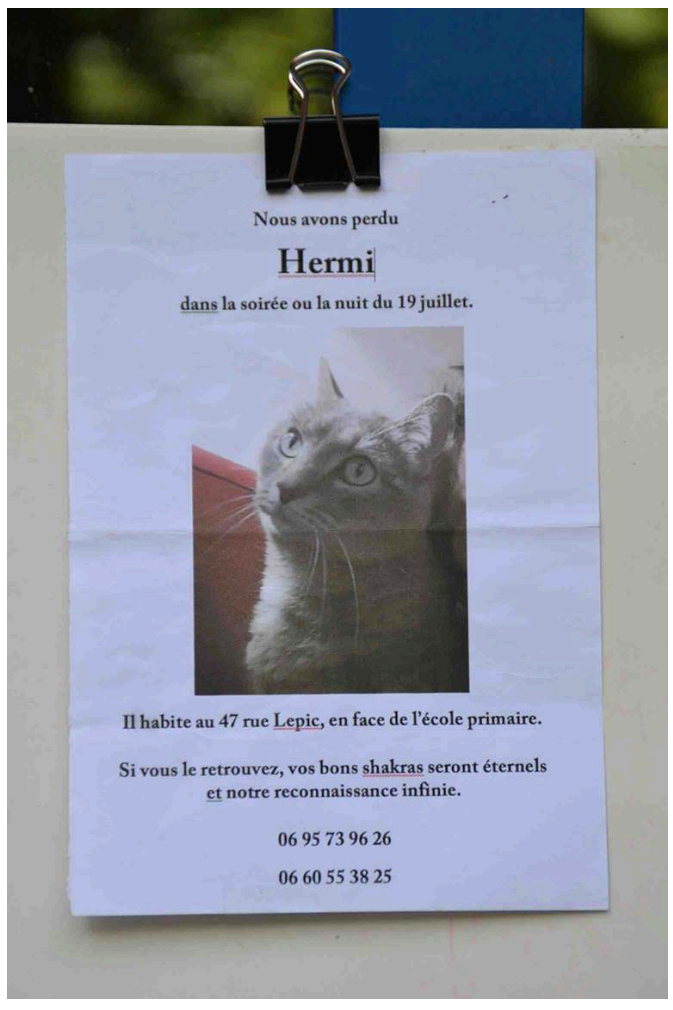

Figure 16. Affichette 2.2. Gino

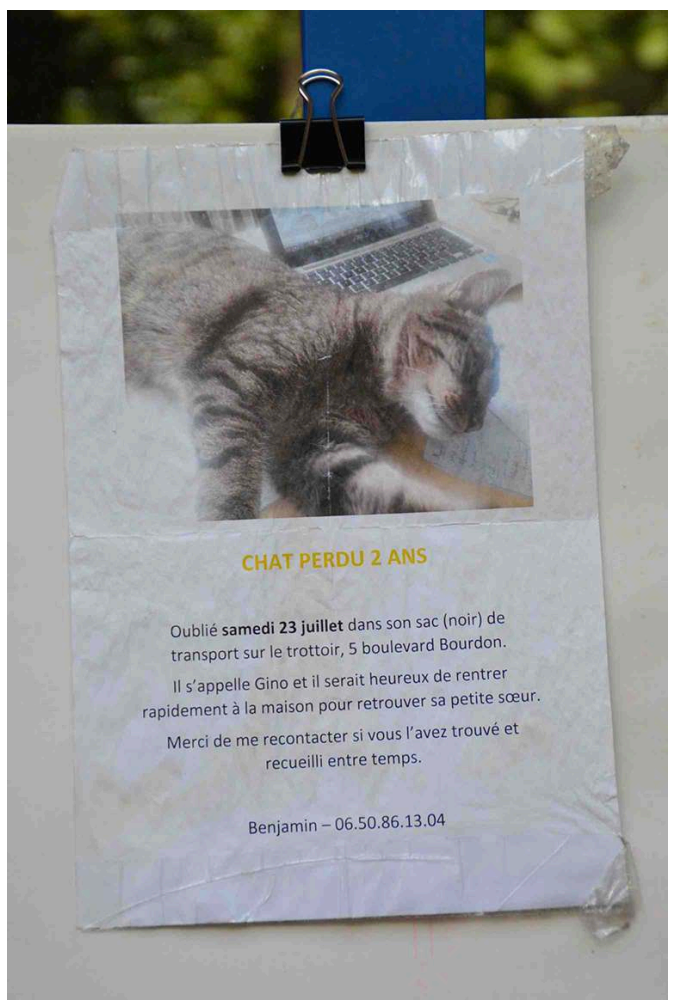




\subsubsection{Anthroponymie animale}

Enfin, la troisième catégorie rassemble le reste des affichettes, 24 sur 32, qui présentent le coefficient d'agentivité le plus faible : l'anthroponymie. Nous y avons rassemblé les affichettes contenant des noms d'animaux qui semblent humains, et qui témoignent donc de l'attribution aux animaux d'une agentivité analogue à celle de l'humainee, via un anthropomorphisme classique. Les anthroponymes deviendraient des zoonymes. Les noms sont les suivants :

- chats : Freddy, Ramsès, Charlie, Maurice, Prospérine, Paula, Willow, Julia, Moïse, Gatsby, Joris (Jojo), Mickey, Helmut, Louis, Diego, Johnny (auxquels s'ajoutent Ellie, Kaye, Sancho, Gino et Simone des catégories 1 et 2);

- chiens : Liam, Tarzan, Jack, Logan, Inès ;

- perroquet : Léon.

Ce phénomène nous a semblé saillant, et porteur de signification. Mais en fait, cette hypothèse de la spécificité humaine de certains prénoms donnés comme noms aux animaux s'avère faible voire inexacte, comme le montre Baptiste Coulmont dans un article sur « le partage des prénoms entre hommes et chiens » (Coulmont 2016). «Entre humains et chiens, écrit-il, si les frontières sociales sont indéniables, il existe une zone frontalière dynamique, assez large et floue, une zone de partage » (2016:159). Il s'agit d'un "entre-deux où le prénom est à la fois canin et humain, mais plus ou moins fortement canin, plus ou moins fortement humain » (2016: 159). En croisant le fichier des prénoms de l'INSEE et le fichier d'identification des chiens, Baptiste Coulmont montre en effet que le prénom humain pour le chien est banal :

Le constat principal est qu'un tiers des chiens portent des prénoms humains. Il faut donc revisiter à nouveaux frais ce qu'écrivait Claude Lévi-Strauss à propos des "chiens, auxquels on ne donne pas de prénom humain sans provoquer un sentiment de malaise, sinon même un léger scandale » (Lévi-Strauss, 2002, 245). Car certains prénoms humains sont bien donnés aux chiens sans aucun malaise, et assez fréquemment. Les données présentes dans le fichier d'identification des chiens permettent de plus, et c'est leur intérêt, de ne pas considérer d'emblée que les prénoms donnés aux humains sont toujours-déjà des prénoms humains. (Coulmont $2016: 159)$

La conclusion est intéressante : non seulement l'identité des prénoms entre humain'es et chien'ne's est courante, mais de plus, il n'est pas sûr qu'il existe une spécificité humaine des prénoms. Baptiste Coulmont montre même que, historiquement, « ce sont les bébés qui reçoivent des "vieux prénoms de chiens", et non pas le contraire » (2016: 163).

Voilà de quoi contribuer à faire descendre l'humaine de son piédestal et à redessiner la pyramide des règnes. Dans notre perspective, si l'on élargit ce travail aux chats et autres animaux, l'identité zoo-anthroponymique prend un autre aspect : au lieu d'être une originalité saillante marquée par l'anthropomorphisme, elle est en fait une norme qui dit la porosité des règnes humain et animal et contredit leur distinction rigide. En ce sens les affichettes d'animaux perdus ne font que formuler et inscrire dans le paysage social urbain la relation que les animaux entretiennent avec nous. 


\section{Conclusion. Des vertus de l'anthropomorphisme}

L'anthropomorphisme, qui nous met si «mal à l'aise» depuis que les animaux ont disparu de notre environnement, relégués dans les zoos et les jouets (Berger 2011), peut être considéré au contraire comme une porte d'entrée dans le monde animal. Il est vain en effet de contester l'attribution aux animaux d'une faculté langagière, culturelle et cognitive en dénonçant une projection anthropomorphique illusoire: retrouver les facultés humaines dans les animaux est en effet le seul moyen que nous ayons d'entrer dans leur univers. C'est ce que ce bref travail a voulu montrer, à partir des affichettes d'animaux perdus, qui, les faisant parler et vivre, ratifient leur agentivité, leur capacité à la perception et leur environnement culturel.

47 Ces affichettes disent que les animaux sont des sujets, et l'on pourrait confirmer cette lecture en explorant par exemple les tombes d'animaux. Au cimetière d'Asnières, premier cimetière pour animaux créé en France en 1899 (il en existe une trentaine actuellement dans l'ensemble du pays), on est frappé par les épitaphes en tous points analogues à celles des tombes humaines : manifestations de douleur, d'amour, lexique familial ou conjugal.

Figure 17. Tombe d'Ophélie, cimetière animalier d'Asnières, 2011.

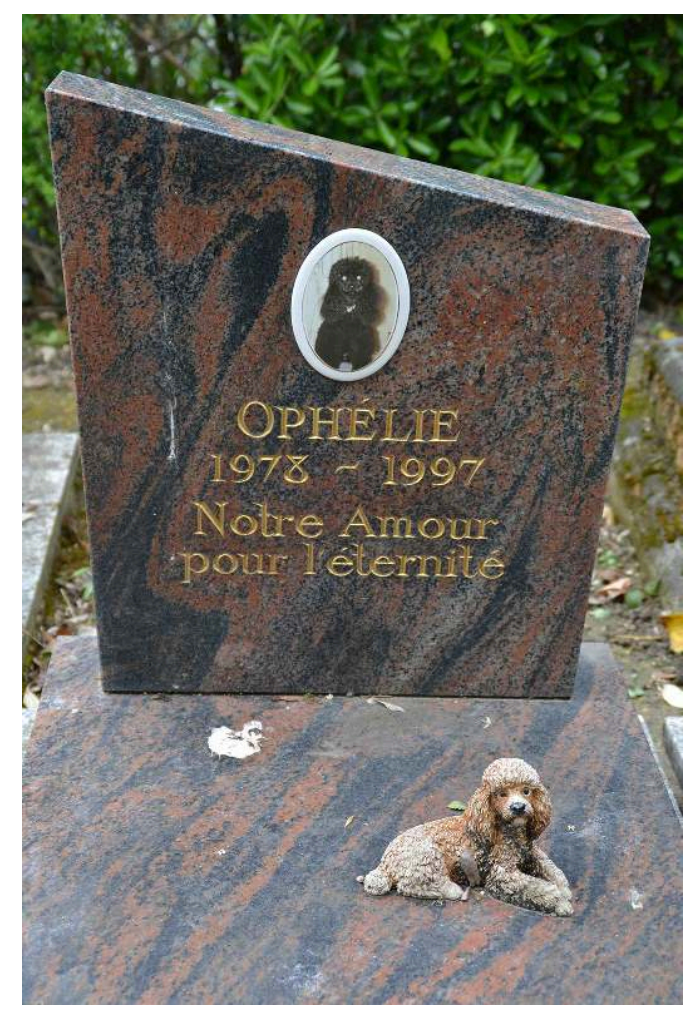


Figure 18. Tombe de Youpi, cimetière animalier d'Asnières, 2011.

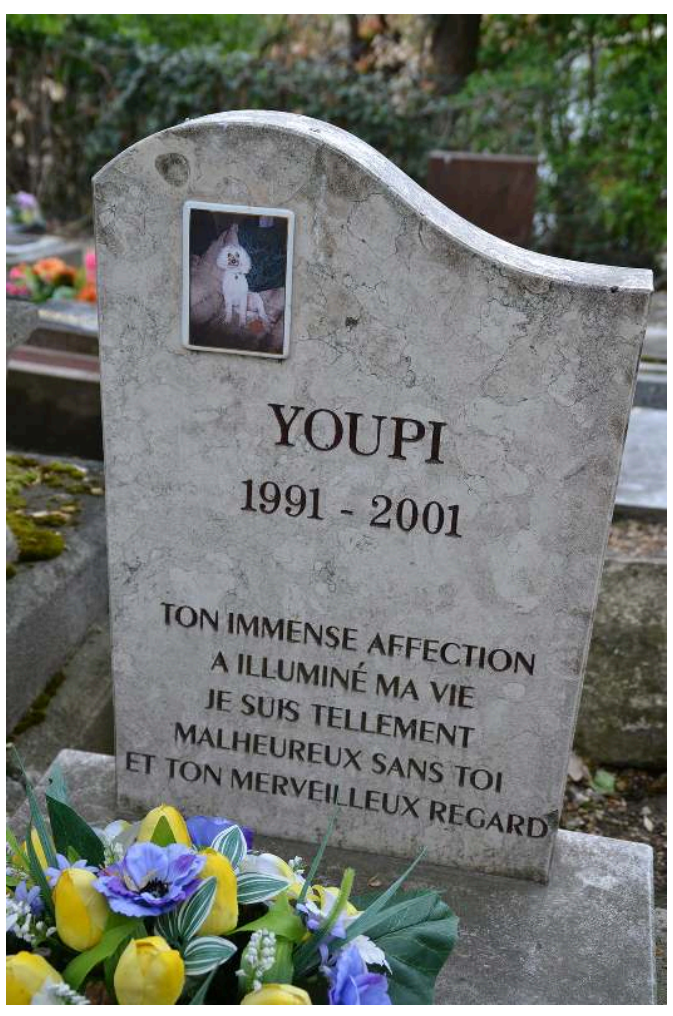

Comme sur les affichettes, les humainee's y formulent une relation, celle qui lie, souvent profondément et durablement, des êtres vivants rassemblés par leur appartenance à l'existence.

\section{BIBLIOGRAPHIE}

Appadurai, Arjun (dir.), [1986] 2009, « Les marchandises et les politiques de la valeur », trad. JeanPierre Warnier, Sociétés politiques comparées, $\mathrm{n}^{\circ} 11, \mathrm{p}$. 3-64.

Berger, John, [2009] 2011, Pourquoi regarder les animaux ?, trad. Katia Berger Anne Andreadakis, Michel Fuchs, Mireille Gouaux et Martin Richet, Genève, Éditions Héros-Limite.

Blanchard, Christophe, 2015, « Ce que les noms des chiens des sans-abris révèlent de leurs maîtres ", Anthropozoologica, n 50-2, p. 99-107.

Butler, Judith, 2004, Le pouvoir des mots. Politique du performatif, trad. Charlotte Nordmann, Paris, Éditions Amsterdam.

Buytendijk, Frederik, 1952, Traité de psychologie animale, trad. Albert Frank-Duquesne, Paris, PUF. Coulmont, Baptiste, 2016, « Des prénoms qui ont du chien : le partage des prénoms entre hommes et chiens ", Annales de démographie historique, $\mathrm{n}^{\circ}$ 131, p. 151-170. 
Danino, Charlotte (dir.), 2018, Les petits corpus, dossier de la revue Corpus, [En ligne], https:// journals.openedition.org/corpus/3094.

De Waal, Frans, 2018, La dernière étreinte. Le monde fabuleux des émotions animales et ce qu'il révèle de nous, trad. Cécile Dutheil de La Rochère, Paris, Les liens qui libèrent.

Daston, Lauren (dir.), 2000, Biographies of Scientific Objects, Chicago, The University of Chicago Press.

Hennion, Antoine et Latour, Bruno, 1993, « Objet d'art, objet de science. Note sur les limites de l'anti-fétichisme ", Sociologie de l'art, $n^{\circ}$ 6, p. 7-24, [En ligne, version auteur], https://

halshs.archives-ouvertes.fr/halshs-00193276.

Lestel, Dominique, 2001, Les Origines animales de la culture, Paris, Flamarion.

Mitchell, William J. Thomas, 1994, Picture Theory: Essays on Verbal and Visual Representation, Chicago, The University of Chicago Press.

Nerlich, Michael, 1990, « Qu'est-ce qu'un iconotexte ? Réflexions sur le rapport texte-image photographique dans La Femme se découvre d'Évelyne Sinnassamy », dans A. Montandon (dir.), Iconotextes, Paris, Ophrys, p. 255-302.

Paveau, Marie-Anne, 2012, «Ce que disent les objets. Sens, affordance, cognition », dans Synergies Pays de la Baltique, $\mathrm{n}^{\circ}$ 9, p. 53-65, [En ligne], http://ressources-cla.univ-fcomte.fr/gerflint/ Baltique9/baltique9.html.

Paveau, Marie-Anne, 2017, L'analyse du discours numérique. Dictionnaire des formes et des pratiques, Paris, Hermann.

Stafford, Andy, 2010, Photo-texts: Contemporary French Writing of the Photographic Image, Liverpool, Liverpool University Press.

Uexküll, Jakob von, [1934] 1965, Mondes animaux et monde humain suivi de Théorie de la signification, trad. Philippe Muller, Paris, Denoël.

\section{NOTES}

1. On trouvera l'ensemble de la sélection, feuilletable grâce à la fonction diaporama, à cette adresse: https://www.dropbox.com/sh/ws3sm4wua46x3dx/ AABake7B7oanaJyopTiRuwF2a?dl=0.

2. Cette réserve sera expliquée plus bas.

3. Les photographies, y compris celles du cimetière d'Asnières dans la conclusion, ont été prises par Marie-Anne Paveau.

4. http://www.alerte-animaux-perdus.fr/saisie-alerte-chien-chat-perdu.php.

5. https://www.rep-secretariat.fr/avis-de-recherche-animal-perdu.

6. https://www.chat-perdu.org/fr-fr/affiche.

7. J'emploie petit corpus au sens où l'expression est envisagée dans un récent numéro de la revue Corpus dédiée à la linguistique de corpus : «Les petits corpus / Small Corpora » (Danino dir. 2018). Les critères minimaux du corpus sont réunis ici; il s'agit d'un classement des données par catégories linguistiques. 


\section{RÉSUMÉS}

Cet article écrit à quatre mains par une collectionneuse et une linguiste assemble une description ethnographique profane d'une collection d'affichettes d'animaux perdus et son analyse discursive. Le travail porte sur un jeu de données de 32 éléments sélectionnés dans une collection de 194 affichettes d'animaux perdus recueillies en contexte urbain, dans des villes françaises et étrangères, entre 2015 et 2018. Ce travail fournit d'abord la description subjective de la collection, puis une analyse des affichettes en tant qu'objets matériels, et enfin son analyse discursive, sous un angle à la fois énonciatif et pragmatique. L'objectif est de montrer que les animaux disposent d'une forme de compétence communicationnelle et surtout d'une agentivité, qui en font des sujets à part entière de la vie sociale.

This paper, written by a collector and a linguist, assembles a secular ethnographic description of a collection of lost animal placards and its discursive analysis. The work focuses on a data set of 32 elements selected from a collection of 194 lost animal placards collected in an urban context, in French and foreign cities, between 2015 and 2018. This work first provides a subjective description of the collection, then an analysis of the placards as material objects, and finally its discursive analysis, from both an enunciative and a pragmatic perspective. The objective is to show that animals have a form of communicative competence and above all an agency, which makes them subjects in their own right in social life.

\section{INDEX}

Keywords : animal agency, animal discursivity, anthropodenial, anthropomorphism, collection, compound statement, phototext, placard

Mots-clés : affichette, agentivité animale, anthropodéni, anthropomorphisme, collection, discursivité animale, énoncé composite, phototexte

\section{AUTEURS}

\section{ELSA ESKENAZI}

Collectionneuse

\section{MARIE-ANNE PAVEAU}

Université Sorbonne Paris Nord, Pléiade (ER 7338) 